Z Rheumatol 2016 $\cdot 75: 13-21$

DOI 10.1007/s00393-015-0033-7

Online publiziert: 19. Januar 2016

๑) Springer-Verlag Berlin Heidelberg 2016

Redaktion

H.-P. Tony, Würzburg

CrossMark

\author{
M. J. Waldner $\cdot$ A. Strangfeld ${ }^{2} \cdot$ M. Aringer \\ 'Medizinische Klinik 1, Universitätsklinikum Erlangen, Erlangen, Deutschland \\ ${ }^{2}$ Deutsches Rheumaforschungszentrum, Berlin, Deutschland \\ ${ }^{3}$ Medizinische Klinik und Poliklinik III, Universitätsklinikum Carl Gustav Carus an der TU Dresden, Dresden, \\ Deutschland
}

\title{
Einfluss der Therapie auf das Krebsrisiko bei rheumatoider Arthritis
}

\section{Pathophysiologische Grundlagen}

\begin{abstract}
Konventionelle, v. a. aber biologische "disease-modifying antirheumatic drugs" (DMARDs) und Immunsuppressiva beeinflussen das Immunsystem deutlich. Damit stellt sich die Frage, wie weit wir durch diese Therapien das Risiko von Malignomen beeinflussen. Immerhin konnte in den vergangenen Jahrzehnten eine zentrale Rolle des Immunsystems bei der Tumorpathogenese gezeigt werden.
\end{abstract}

Zum einen verdeutlicht die erhöhte Inzidenz verschiedener Tumorarten bei Immunschwäche die zentrale Bedeutung des Immunsystems bei der Tumorabwehr [8], zum anderen stellten zahlreiche epidemiologische Studien eine Assoziation verschiedener Infektionen oder chronischer Entzündungen mit bestimmten Tumorarten fest und postulierten eine Förderung der Tumorentstehung durch entzündliche Erkrankungen [4]. Arbeiten aus der Grundlagenforschung ermöglichen die Identifikation molekularer Signalwege, die an diesen Vorgängen beteiligt sind.

In der Onkologie hat ein besseres Verständnis der Manipulation des Immunsystems durch Tumoren zu ersten Ansätzen einer Tumortherapie durch gezielte Modulation des Immunsystems geführt. Umgekehrt kann eine immunmodulatorische Therapie bei entzündlichen Erkrankungen, wie z. B. rheumatischen Erkrankungen, das Risiko für ein erst- maliges Auftreten oder ein Rezidiv einer Tumorerkrankung beeinflussen.

Der folgende Artikel gibt einen Überblick über aktuelle Konzepte zur Bedeutung des Immunsystems bei der Krebsentstehung. Dabei wird besonders auf Signalwege eingegangen, die im Rahmen aktueller Therapieformen rheumatischer Erkrankungen beeinflusst werden.

\section{Allgemeine Aspekte der Tumorentstehung}

Im Jahr 2000 veröffentlichten Hanahan und Weinberg [11] mit den „Hallmarks of cancer" 6 verschiedene Eigenschaften, die für die Entstehung maligner Tumoren zwingend nötig sind. Dazu gehören

- die Unabhängigkeit von Tumorzellen gegenüber exogenen Wachstumsfaktoren, also die Fähigkeit, trotz niedriger Wachstumsfaktorkonzentrationen zu proliferieren,

- eine Resistenz gegen Apoptose,

- eine Unempfindlichkeit gegenüber wachstumshemmenden Faktoren,

- ein unendliches Reproduktionspotenzial,

- eine Aufrechterhaltung der Sauerstoff- und Nährstoffversorgung durch Stimulation der Angiogenese sowie

- invasives und metastasierendes Wachstum.

Während der Tumorentstehung treten diese Veränderungen nach und nach auf, wobei die zeitliche Reihenfolge keine Rolle spielt.

Grundlage für die Entstehung der Veränderungen in tumorrelevanten $\mathrm{Si}$ gnalwegen sind genetische (z. B. Punktmutationen, chromosomale Aberration etc.) oder epigenetische Veränderungen (z. B. Methylierung) von Onkogenen. Diese führen entweder durch einen Funktionsgewinn ("gain of function“, GOF) zur erhöhten Aktivität tumorfördernder Mechanismen oder über einen Funktionsverlust („loss of function“, LOF) von Tumorsuppressorgenen $\mathrm{zu}$ einem reduzierten Schutz gegen die Tumorentstehung.

Ursache der Mutationen sind DNAVeränderungen, die sowohl durch Umweltfaktoren (UV-Strahlung, chemische Substanzen etc.) als auch durch körpereigene Faktoren (reaktive Sauerstoffspezies, Hydrolyse etc.) bedingt werden können. DNA-Veränderungen werden auch durch therapeutische Maßnahmen induziert. Das gilt insbesondere für die Strahlentherapie und die meisten Formen der Chemotherapie. Unter den in der Rheumatologie heute üblichen Medikamenten kommt dafür nur Cyclophosphamid infrage, das als alkylierendes Zytostatikum direkt DNA-Schäden induziert. Cyclophosphamid kann nicht nur Malignome im ableitenden Harnwegsbereich induzieren, sondern erhöht dosisabhängig auch das Risiko akuter myeloischer Leukämien [7]. Beide Arten von Malignomen treten oft mit jahrzehntelanger Latenz 


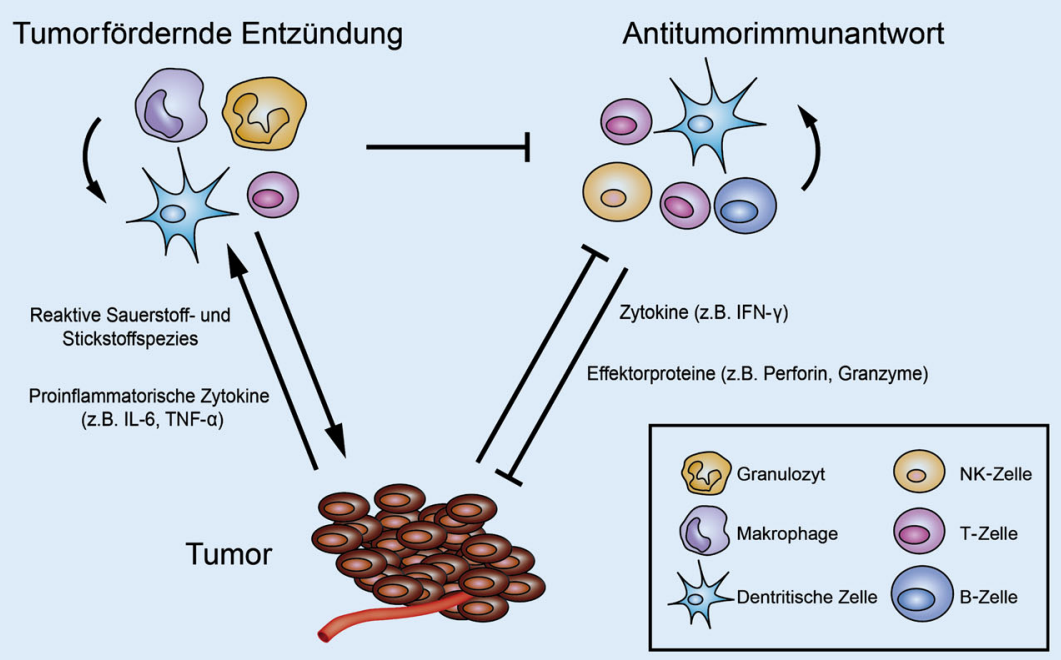

Abb. 1 A Duale Rolle des Immunsystems bei der Tumorpathogenese. Im Rahmen chronisch entzündlicher Erkrankung werden die Tumorentstehung und das Tumorwachstum durch die Freisetzung reaktiver Sauerstoff- und Stickstoffspezies sowie durch proinflammatorische Zytokine gefördert. Im Gegensatz dazu hemmt die Antitumorimmunantwort die Tumorprogression v. a. durch das adaptive Immunsystem mit Freisetzung von Effektorproteinen und Zytokinen, wie z. B. Interferon- $\gamma$ (IFN- $\gamma$ ). (Abbildung adaptiert nach [9])

nach der Cyclophosphamidtherapie auf. Dieses Risiko ist für die anderen Immunsuppressiva, Methotrexat (MTX) und die anderen konventionellen DMARDs, aber auch für die Biologika auf Basis der Mechanismen und der epidemiologischen Daten nicht anzunehmen.

\section{》) Ursache der Tumorentste- hung sind DNA-Veränderungen}

Da DNA-Schäden im menschlichen Körper relativ häufig auftreten, existieren sehr effektive Schutzprogramme zur Erkennung und Reparatur von DNA-Schäden und zur Abtötung stark geschädigter Zellen [13]. Diese Abwehrsysteme verhindern in der Regel, dass in einer Zelle eine Anhäufung mehrerer Mutationen auftritt, die zu den 6 oben genannten „Hallmarks of cancer" führt. Daher geht man davon aus, dass für die Krebsentstehung ein verändertes Verhältnis zwischen der Häufigkeit von DNA-Schäden und der Effizienz der Abwehrmechanismen nötig ist (๑Abb. 1). Interessanterweise zeigen v. a. Forschungsarbeiten der letzten Jahre, dass das Immunsystem sowohl vermehrte DNA-Schäden induziert als auch an der Abwehr der Konsequenzen beteiligt ist - und somit eine maßgebliche Rolle bei der Krebsentstehung und gleichzeitig bei der Krebsabwehr spielt.

\section{Tumorfördernde Effekte chronischer Entzündungen}

Das erhöhte Risiko bestimmter Krebserkrankungen bei chronischen Infektionen und Entzündungsprozessen führte zu dem Konzept einer Förderung der Krebsentstehung durch das Immunsystem. Bekannte Beispiele sind das erhöhte Risiko für Magenkarzinome bei einer Helicobacter-pylori-Infektion, für kolorektale Karzinome bei chronisch entzündlichen Darmerkrankungen, für hepatozelluläre Karzinome bei chronischer Virus-Hepatitis B oder C, für Zervixkarzinome bei HPV-Infektion, für Osteosarkome bei Morbus Paget oder Narbenkarzinome [29].

Grundlagenarbeiten konnten zeigen, dass die Entzündungsreaktion v. a. durch Aktivierung des angeborenen Immunsystems - an nahezu allen Schritten der Tumorentstehung beteiligt ist [14]. Diese werden unterteilt in die als Initiierung bezeichnete Entartung einzelner Zellen durch genetische oder epigenetische Veränderungen, die Tumorpromotion, die eine anhaltende
Proliferation entarteter Zellen darstellt, und schließlich die Progression, bei welcher der Tumor maligne Kriterien wie infiltratives Wachstum und Metastasierung entwickelt.

\section{》) Erhöhter oxidativer Stress führt zu DNA-Schäden}

Hinsichtlich der Initiierung geht man davon aus, dass v. a. ein erhöhter oxidativer Stress zu DNA-Schäden führt. Dabei setzen in das Gewebe einwandernde Granulozyten und Makrophagen u. a. reaktive Sauerstoff- und Stickstoffspezies frei, die zu Einzel- und Doppelstrangbrüchen und Nukleotidveränderungen in der DNA führen können. Wenn diese DNA-Veränderungen zufällig Onkogene betreffen, ist der Grundstein für die Tumorentstehung gelegt.

Anschließend wird die zunehmende Proliferation der initiierten Tumorzellen im Rahmen der Tumorpromotion nicht nur durch klassische Wachstumsfaktoren, sondern auch durch proinflammatorische Zytokine beeinflusst. So konnte $z$. B. für Tumornekrosefaktor- $\alpha$ (TNFa), Interleukin-6 (IL-6) und IL-22 ein direkter proliferationsfördernder und antiapoptotischer Effekt auf Tumorzellen nachgewiesen werden [9].

Diese Effekte werden in den Tumorzellen durch die Aktivierung von Signalwegen vermittelt, die den Zellzyklus direkt beeinflussen oder durch Transkriptionsfaktoren wie NF- $\kappa B$ („nuclear factor ,kappa-light-chain-enhancer ${ }^{r}$ of activated B cells“) oder STAT3 („,ignal transducer and activator of transcription $3^{\prime \prime}$ ) zu einer Veränderung der Genexpression führen. Diese veränderte Genexpression kann wiederum zu einer Veränderung der Zellfunktion mit Entwicklung eines malignen Phänotyps führen.

In diesem Zusammenhang ist einerseits interessant, dass sich die Signalwege von Wachstumsfaktoren und Zytokinen zumindest teilweise überlappen. Andererseits stellen Mutationen bestimmter Signalmoleküle, wie z. B. von STAT3 (dem wichtigsten Signaltransduktionsmolekül von IL-6), selbst onkogene Mutationen dar. 
Hier steht eine Anzeige.

算 Springer 
Ein großer Teil der Mechanismen, die an der entzündungsabhängigen Tumorentstehung beteiligt sind, ist bei der Wiederherstellung eines Gewebeschadens von zentraler Bedeutung. Daher wurde diese Art der Tumorentstehung mit einer überschießenden Wundheilung verglichen.

Die Mechanismen der entzündungsabhängigen Tumorentstehung werden auf molekularer Ebene nur in Grundzügen verstanden. Ihre klinische Relevanz wird aber durch die Beobachtung gestützt, dass eine suffiziente und dauerhafte Therapie der Grunderkrankung (chronische Entzündung oder Infektion) zu einer deutlichen Verringerung des erhöhten Tumorrisikos führen kann. So wurde in den vergangenen Jahrzehnten ein Rückgang der kumulativen Inzidenz für kolorektale Karzinome bei Colitis ulcerosa von über 30/1000 Patientenjahre auf unter 10/1000 Patientenjahre beschrieben, der sich am ehesten durch eine verbesserte Behandlung der intestinalen Entzündung erklären lässt [3].

\section{Lymphomrisiko bei Autoimmun- erkrankungen}

Bei vielen Autoimmunerkrankungen, unter anderem vielen entzündlich rheumatischen Erkrankungen, wurde ein erhöhtes Lymphomrisiko nachgewiesen [6]. Da dieses Risiko mit der Aktivität der Erkrankung steigt, wurde es zunächst in mehreren Fällen neuen Therapien zugeordnet, die typischerweise bei den Patienten mit besonders aktiver Autoimmunerkrankung verwendet werden. Unter anderem standen die Therapien mit MTX oder TNF-Blockern im Verdacht, zur Entstehung von Lymphomen zu führen. Sobald aufgrund größerer Datensätze neben der Therapie auch die Krankheitsaktivität in die Berechnungen einbezogen werden konnte, erschien das erhöhte Lymphomrisiko durchweg aktivitätsassoziiert [2].

\section{》) Erhöhtes Lymphomrisiko ist aktivitätsassoziiert}

Dafür werden 3 verschiedene Mechanismen diskutiert. Erstens erhöht die chro-

Z Rheumatol 2016 · 75:13-21 DOI 10.1007/s00393-015-0033-7

c) Springer-Verlag Berlin Heidelberg 2016

\section{J. Waldner $\cdot$ A. Strangfeld $\cdot$ M. Aringer}

\section{Einfluss der Therapie auf das Krebsrisiko bei rheumatoider Arthritis. Pathophysiologische Grundlagen}

\section{Zusammenfassung}

Die erhöhte Inzidenz bestimmter Tumorarten im Rahmen chronischer Entzündungen und Infektionen einerseits und das erhöhte Tumorrisiko unter Immunsuppression andererseits verdeutlichen die vielseitige und komplexe Beeinflussung der Tumorpathogenese durch das Immunsystem. Daher ist gut vorstellbar, dass immunmodulatorische Therapien neben der Grunderkrankung auch das Malignomrisiko beeinflussen. Arbeiten aus der Grundlagenforschung identifizierten verschiedene immunologische Signalwege, die für die Tumorentstehung bzw. Tumorabwehr relevant sind. Diese Übersichtsarbeit stellt aktuelles Wissen über Effekte des Immunsystems auf die Krebsentstehung dar und versucht, soweit dies möglich ist, das Krebsrisiko unter den vorhandenen Therapieoptionen zu konkretisieren.

\section{Schlüsselwörter}

Disease-modifying anti-rheumatic drugs . Tumorpathophysiologie · Immunsuppression . Immunsystem · Entzündung

\section{Influence of therapy on risk of cancer in rheumatoid arthritis. Pathophysiological principles}

\section{Abstract}

The increased incidence of certain tumor entities in chronic inflammation and infections as well as the increased tumor risk under immunosuppression, illustrate the multiple and complex influences of the immune system on tumor pathogenesis. It is therefore conceivable that immunomodulatory therapy for rheumatoid arthritis (RA) could also influence the risk of malignancies, apart from treating the underlying disease. Basic scientific research studies have identified various signal transduction pathways that are relevant for tumorigenesis and tumor defense mechanisms. This review presents the current knowledge on the effects of the immune system on the occurrence of cancer and tries, where possible, to relate it to specific treatment options.

\section{Keywords}

Disease-modifying antirheumatic drugs · Immunosuppression - Tumor pathophysiology . Immune system · Inflammation nische Proliferation von Lymphozyten im Rahmen der Autoimmunerkrankung per se das Risiko einer malignen Entartung. Schließlich bedeutet jede Zellteilung ein Risiko für Fehler. Zweitens stellen Zytokine im Immunsystem auch nur einen Sonderfall von Überlebensfaktoren dar. So geben Zytokine wie IL-2, IL-7, IL15 , aber auch BLyS/BAFF bestimmten Lymphozytenpopulationen ein oft notwendiges Überlebenssignal. Dieses Signal gibt dem Immunsystem normalerweise die Möglichkeit, das Immunrepertoire zu erweitern, um eine Infektion erfolgreich zu bekämpfen. Es erhöht aber auch die Chance transformierter Lymphozyten, weiter zu überleben. Und drittens könnten tumorassoziierte Virusinfektionen durch die Autoimmunität eher gefördert werden. Vielleicht führt der Fokus des Immunsystems auf das Autoantigen eher zum Übersehen anderer Bedrohungen. Zumindest scheint die Häufigkeit von Herpes zoster bei systemischem Lupus erythematodes (SLE) und rheumatoider Arthritis (RA) erhöht.

\section{Tumorrisiko bei chronisch entzündlichen Darmerkrankungen}

Bei beiden Hauptformen chronisch entzündlicher Darmerkrankungen (CED), Morbus Crohn und Colitis ulcerosa, führt die anhaltende Entzündung des Dickdarms zu einem erhöhten Risiko für kolorektale Karzinome (KRK). Das Risiko der Entstehung Kolitis-assoziierter Karzinome ist von der Lokalisation, Ausdehnung, 
Dauer und Ausprägung der intestinalen Entzündung abhängig. So ist z. B. bei der Colitis ulcerosa das Tumorrisiko bei einer Pankolitis am höchsten, während es bei einer Linksseitenkolitis nur geringgradig erhöht ist. $\mathrm{Ob}$ auch die Proktitis mit einem erhöhten Risiko einhergeht, ist bisher nicht gesichert. Insgesamt wird das kumulative Risiko für ein KRK bei Colitis ulcerosa mit 1,6\% nach 10 Jahren, $8,3 \%$ nach 20 Jahren und 18,4\% nach 30 Jahren Erkrankungsdauer angegeben. Für den Morbus Crohn ergab sich ein kumulatives Risiko von 2,9\% nach 10 Jahren, 5,6 \% nach 20 Jahren und 8,3\% nach 30 Jahren Erkrankungsdauer [31].

In Arbeiten zur Aufklärung der gesteigerten Karzinomentstehung bei CED konnten immerhin einzelne Signalwege identifiziert werden, die sowohl an der Pathogenese der CED als auch des damit assoziierten KRKs beteiligt sind. Dabei handelt es sich u. a. um die bereits beschriebenen Mechanismen der Aktivierung von STAT3 in kolorektalen Karzinomzellen durch IL-6 und IL-22 oder von NFkB durch TNF-a. Obwohl eindeutige klinische Daten bisher fehlen, ist die rückläufige Inzidenz des Kolitis-assoziierten Karzinoms in den vergangenen Jahren mit einer wirksamen Prävention durch die adäquate Therapie der intestinalen Entzündung vereinbar.

\section{Tumorrisiko bei rheumatoider Arthritis}

Im Gegensatz zu den chronisch entzündlichen Darmerkrankungen gibt es bei rheumatischen Erkrankungen bisher keine überzeugenden Hinweise für eine aktivitätsassoziierte Häufung epithelialer Tumoren. Das mag der Tatsache geschuldet sein, dass im Gelenkbereich solche Gewebestrukturen kaum vorkommen und Fibroblasten und Chondrozyten nicht zur Entartung neigen. Bei Patienten mit RA gibt es 2 Arten von epithelialen Tumoren, die signifikant erhöht auftreten, nämlich einerseits Lungenkarzinome [26] und andererseits Nicht-Melanom-Hautkrebs [18].

Bei beiden ist ein direkter Zusammenhang mit dem Autoimmungeschehen eher unwahrscheinlich. Während die Assoziation mit dem Lungenkrebs mit der pathogenetischen Bedeutung des Rauchens für beide Erkrankungen zusammenhängen dürfte [17], gibt es für die höhere Rate von Basaliomen und evtl. Plattenepithelkarzinomen der Haut noch keine überzeugende Erklärung. Auch hier scheinen gemeinsame Risikofaktoren, vielleicht auch gemeinsame Risikogene, plausibler als ein Zusammenhang mit der Aktivität der RA. Eine gewisse Rolle könnte auch eine durch DMARDs wie MTX induzierte UVEmpfindlichkeit spielen.

Lymphome, in der Regel Non-Hodgkin-B-Zell-Lymphome, sind hingegen bei der RA und anderen entzündlich rheumatischen Erkrankungen klar und aktivitätsbezogen erhöht [19]. Statistisch lässt sich dieses Risiko durch eine adäquate Krankheitskontrolle mit konventionellen und/oder biologischen DMARDs senken. Das ändert aber nichts daran, dass in Einzelfällen die spontane Regression von Lymphomen nach $\mathrm{Ab}$ setzen von MTX, aber auch von TNFBlockern eine mögliche pathogenetische Rolle dieser Substanzen im Sonderfall denkbar erscheinen lässt [24].

\section{Tumorabwehr durch das adaptive Immunsystem}

Ähnlich wie tumorfördernde Effekte chronischer Entzündungen herausgearbeitet wurden, gibt es auch klinische Beispiele für ein erhöhtes Tumorrisiko bei Immundefizienz. Die wahrscheinlich am besten untersuchten Beispiele sind Aids und die medikamentöse Immunsuppression nach Organtransplantation. In beiden Fällen besteht ein deutliches erhöhtes Risiko für virusassoziierte Tumorerkrankungen wie Morbus Hodgkin und Non-Hodgkin-Lymphome bei EBV (Epstein-Barr-Virus)-Infektion, HHV (humane Herpesvirus)-8-assoziiertes Kaposi-Sarkom und HBV (Hepatitis-BVirus)- und HCV (Hepatitis-C-Virus)assoziierte hepatozelluläre Karzinome [10]. Unabhängig von bekannten Infektionen wurde auch über ein erhöhtes Risiko für Nicht-Melanom-Hautkrebs und Lippenkarzinome, solide Malignome und Leukämien berichtet. Auch hier werden jedoch Viruserkrankungen als mögliche Ursachen diskutiert. Hingegen scheint das Risiko für Adenokarzinome wie kolorektale Karzinome, Mammakarzinom, Ovarialkarzinom und Prostatakarzinom nicht erhöht zu sein.

Während man bei den infektionsassoziierten Tumorerkrankungen primär von einer unzureichenden Kontrolle der Infektion durch das adaptive Immunsystem und erst sekundär von direkten tumorfördernden Effekten der Erreger und der Entzündungsreaktion ausgeht, gibt es verschiedene Konzepte bezüglich der nicht infektionsassoziierten Tumorerkrankungen. Zwar kann nicht ausgeschlossen werden, dass auch diese Tumorarten durch bisher unbekannte Infektionen hervorgerufen werden, aber sowohl pathologische Assoziationen als auch Grundlagenarbeiten geben Hinweise auf eine relevante Antitumorimmunantwort durch das adaptive Immunsystem, die bei Immunsuppression eingeschränkt ist.

Pathohistologisch ist eine Infiltration des Tumorgewebes mit verschiedenen Zellen des adaptiven Immunsystems bei zahlreichen soliden Tumorarten mit einer verbesserten Krankheitsprognose assoziiert. So korreliert eine erhöhte Infiltration von $\mathrm{CD}^{+}{ }^{+} \mathrm{CD} 45 \mathrm{RO}^{+}-\mathrm{T}$-Zellen oder Th1-Zellen in Mammakarzinome, Ovarialkarzinome, Ösophaguskarzinome, kolorektale Karzinome, Bronchialkarzinome oder Melanome mit einem besseren progressionsfreien Überleben und Gesamtüberleben [8]. Diese Zellpopulationen sind wesentlich an der zytotoxischen Immunantwort beteiligt.

Beweisen lässt sich diese Hypothese im Tiermodell. So zeigen Mäuse mit einem Defekt unterschiedlicher Komponenten des adaptiven Immunsystems im Vergleich zu Mäusen ohne diesen Defekt ein erhöhtes Tumorwachstum. Entscheidend sind dabei v. a. Defekte in der Entwicklung und Funktion von zytotoxischen CD8 ${ }^{+}$T-Zellen, CD4 ${ }^{+}$Th1Zellen und natürlichen Killer (NK)-Zellen, die maßgeblich an der Erkennung und anschließenden Zerstörung entarteter Zellen beteiligt sind. Die Infiltration dieser Zellen in den Tumor wurde bei verschiedenen humanen Tumorerkrankungen mit einer verbesserten Prognose assoziiert [28]. 
Tab. 1 Veränderungen, die (schrittweise) zu malignen Tumoren führen

Zunahme maligner Eigenschaften

Erhöhte Proliferation

Unabhängigkeit von Wachstumsfaktoren

Resistenz gegen Steuerungsfaktoren

Verlust der Zellalterung

Neoangiogenese

Überschreiten von Organgrenzen

Verlassen des Zellverbands

Verlust interner Sicherungsprogramme

DNA-Reparatur

Apoptose

Blockade externer Sicherungsprogramme

Immun-Checkpoint-Manipulation

Resistenz gegen induzierte Apoptose

Die Erkennung der entarteten Zellen erfolgt v. a. durch die Detektion veränderter Antigene, die durch Mutationen entstehen. Veränderte Peptide werden dem adaptiven Immunsystem $\mathrm{Ma}$ jor Histocompatibility Complex(MHC)gebunden präsentiert. Eine Erkennung veränderter Antigene führt durch zytotoxische Effektormechanismen zur Zerstörung der veränderten Zelle.

Neben den zellintrinsischen Signalwegen stellt also das Immunsystem einen zweiten Mechanismus zur Abwehr mutierter Zellen dar. Wie bereits bei den zellintrinsischen Abwehrmechanismen kommt die Frage auf, warum trotz dieser Abwehr Tumorerkrankungen auftreten. Um dies zu erklären, unterscheidet man aktuell 3 Phasen bei der Antitumorimmunantwort. In der Eliminationsphase werden die Tumorzellen durch das Immunsystem erkannt und zerstört. Ist die Elimination vollständig, ist das Problem dadurch aus der Welt geschafft. Gelingt es Tumorzellen hingegen, sich an die Immunantwort $\mathrm{zu}$ adaptieren, entsteht zunächst ein Equilibrium zwischen Tumorwachstum und Zerstörung durch das Immunsystem. Treten weitere Veränderungen des Tumors auf, kann es zu einem „Escape“, also einer Umgehung der Immunantwort, kommen; der Tumor zeigt plötzlich eine deutliche Progression [28].

Dabei spielt unter anderem die Aktivierung sog. inhibitorischer CheckpointSignalwege wie des CTLA-4- und des PD-
1-Signalwegs eine Rolle. Diese verhindern unter physiologischen Bedingungen Autoimmunität und kritische Gewebeschäden $z$. B. bei Infektionen. Tumorzellen können sich diese Mechanismen aneignen und damit aktiv das Immunsystem lahmlegen (• Tab. 1).

\section{》) Inhibition der Check- point-Signalwege hemmt Tumorwachstum}

Nachdem in Tiermodellen gezeigt werden konnte, dass eine Inhibition dieser Checkpoint-Signalwege das Wachstum verschiedener Tumorarten hemmt, wurden in den vergangenen Jahren verschiedene klinische Studien mit diesen Medikamenten durchgeführt [30]. Die vielversprechenden Ergebnisse dieser Studien führten zur Zulassung des AntiCTLA-4-Antikörpers Ipilimumab sowie der Anti-PD-1-Antikörper Nivolumab und Pembrolizumab zur Behandlung von metastasierten malignen Melanomen und zeigen damit eine relevante Antitumorimmunantwort bei Patienten.

Die Tatsache, dass CTLA-4-Antagonisten bei Tumorerkrankungen erfolgreich eingesetzt werden können, CTLA4-Agonisten jedoch bei Autoimmunerkrankungen wie auch der RA zum Einsatz kommen, verdeutlicht die teilweise gegenläufige Bedeutung des Immunsystems bei beiden Erkrankungsformen. In diesem Zusammenhang werden im Anschluss die wichtigsten molekularen $\mathrm{Si}$ gnalwege, die bei der Therapie der RA beeinflusst werden, hinsichtlich einer Beeinflussung des Krebsrisikos einzeln diskutiert.

\section{Effekte zellspezifischer biologischer DMARDs}

\section{CD28-Antagonist Abatacept: Hemmung der T-Zell-Aktivierung}

Abatacept, CTLA-4-Ig, blockiert CD80 und CD86, die Liganden von CD28. Damit werden die Kostimulation über CD28 und in der Folge die T-Zell-Aktivierung verhindert. Daraus lässt sich der lang anhaltende Effekt von Abatacept [21] erklären, aber nicht sein unerwartet schneller
Wirkungseintritt [22]. Zudem sprechen murine Daten für einen T-Zell-unabhängigen Effekt [15], der nach wie vor nicht geklärt ist.

Die Tatsache, dass der Antikörper Ipilimumab, der den endogenen Kostimulationshemmer CTLA-4 blockiert, Antitumoreffekte zeigt [12], führt zur unmittelbaren Frage, ob die Abatacept-Therapie das Tumorrisiko erhöht. Während in frühen klinischen Phasen der Abatacept-Entwicklung Lungentumoren zunächst etwas gehäuft schienen, hat sich das aber als Ergebnis statistischen Zufalls herausgestellt. Aus heutiger Sicht besteht kein belastbarer Anhalt für eine Häufung solider Malignome unter Abatacept [25, 33]. Bisher gibt es auch keinen Beleg für ein erhöhtes Melanomrisiko unter Abatacept. Das mag aber an der noch immer begrenzten Datenmenge für den Kostimulationsblocker liegen.

\section{Anti-CD20-Antikörper Rituximab: B-Zell-Depletion}

Der chimärische monoklonale Antikörper Rituximab bindet das B-Zellspezifische Antigen CD20. Rituximab führt hauptsächlich zu einer Depletion von B-Zellen, die im peripheren Blut typischerweise nahezu vollständig, im Gewebe aber etwas variabler ist und über viele Monate anhält. Nicht betroffen sind B-Zell-Vorstufen und Plasmazellen, sodass auch die Immunglobuline meist nicht relevant absinken. Auch die therapeutischen Effekte werden meist nicht oder nur unwesentlich durch einen direkten Effekt auf die Bildung von $\mathrm{Au}$ toantikörpern verursacht. Sie beruhen annehmbar auf dem Ausfall von B-Zellen als hoch effizienten, spezifischen Antigen präsentierenden Zellen und möglicherweise auch auf der Reduktion der Zytokinfreisetzung durch B-Zellen.

Für B-Zell-Lymphome, in deren Therapie Rituximab und seine Folgesubstanzen eine wesentliche Rolle spielen, war ein erhöhtes Risiko nicht zu erwarten. In einem nur teilweise begründeten Analogieschluss wurde Rituximab nach Tumorerkrankungen als am ehesten vertretbares biologisches DMARD gesehen. Tatsächlich gibt es mittlerweile epidemiologische Argumente für diesen Zugang: Obwohl 
Hier steht eine Anzeige.

算 Springer 
es aus der Grundlagenforschung Hinweise für eine Beteiligung von B-Zellen an der Antitumorimmunantwort gibt [34], haben sich in den nun fast 10 Jahren der Anwendung von Rituximab bei RA keine Hinweise für ein erhöhtes Malignomrisiko ergeben. De facto war in der Kohorte des deutschen Biologika-Registers RABBIT das Rezidivrisiko - trotz des Einschlusses einer Population mit höherem Risiko - unter Rituximab, wenn auch statistisch nicht signifikant, tendenziell niedriger als unter anderen biologischen DMARDs, insbesondere TNF-Blockern [27].

\section{Effekte zytokinspezifischer biologischer DMARDs}

\section{Anti-IL-6-Antikörper Tocilizumab: Hemmung proinflammatorischer Signalwege}

Als erster Inhibitor des IL-6-Signalwegs wurde 2009 der Anti-IL-6-Rezeptor-Antikörper Tocilizumab zugelassen. Über die Bindung an einen membrangebundenen oder löslichen IL-6-Rezeptor kann IL-6 die Aktivierung eines trimeren Rezeptors mit 2 Molekülen der (auch zu anderen Rezeptoren gehörenden) Rezeptorkette gp130 (CD130) induzieren und über die Januskinase 1 den Transkriptionsfaktor STAT3 aktivieren. STAT3 führt zur Transkription unterschiedlicher Zielgene, die sowohl an der akuten Entzündungsreaktion als auch an der Wiederherstellung des Gewebes im Rahmen der Wundheilung beteiligt sind.

Aufgrund der zahlreichen IL-6-Effekte und der bereits nachgewiesenen Beteiligung an verschiedenen entzündungsabhängigen Tumorarten wäre eigentlich die Hypothese aufzustellen, dass eine Hemmung eines chronisch erhöhten IL-6 das Risiko für Adenokarzinome und Lymphome nicht erhöhen, sondern eher verringern sollte. Andererseits ist IL-6 ein antiapoptotisches Zytokin für T-Zellen und fördert die Proliferation von B-Zellen. Gerade in der Phase der frühen Malignomentstehung ist daher auch ein negativer Effekt der IL-6-Blockade auf die Immun-Surveillance nicht mit Sicherheit auszuschließen.
Die bisherigen Daten konnten weder ein erhöhtes noch ein reduziertes Karzinomrisiko im Rahmen der TocilizumabTherapie feststellen. Zumindest wurde Tocilizumab aber in Einzelfällen erfolgreich zur Behandlung einer Tumorkachexie eingesetzt [1] und schien in einer Phase-I-Studie bei Patientinnen mit epithelialen Ovarialkarzinomen gewisse Antitumoreffekte zu haben [5]. Zudem ist ein Effekt auf das maligne Myelom bekannt [23].

\section{Anti-TNF-Antikörper: Einfluss nicht nur auf das Immunsystem}

Die Hemmung eines Zytokins, das Tumornekrosefaktor (TNF) heißt, führt bei vielen Patienten zu Sorgen in Bezug auf das Tumorrisiko. TNF hat eine Vielzahl von Effekten, die nach wie vor inkomplett verstanden werden. Primär ist TNF ein proinflammatorisches Zytokin, das über NF- $\mathrm{KB}$ - und MAPK-Signalwege wirkt. Gerade NF- $\kappa B$-induzierte Gene wirken auch gegen die Apoptose, den geplanten, regelrecht ablaufenden Zelltod alter und geschädigter Zellen. Einer der beiden TNF-Rezeptoren, TNF-Rezeptor 1 (CD120a), kann hingegen über die indirekte Assoziation mit Caspasen direkt Apoptose auslösen, sodass auf dieser Ebene prinzipiell beide Möglichkeiten bestehen. Zytotoxische Lymphozyten tragen zum Teil membrangebundenen TNF auf ihrer Oberfläche. Auszuschließen ist hier ein Einfluss auf (frühe) Malignome nicht. Zudem hat TNF eine Reihe systemischer Effekte - von der Auslösung einer Kachexie (ein weiterer Ausdruck für das $\mathrm{Zy}$ tokin war Cachectin) bis zur Erhöhung der Permeabilität von Gefäßen. Insgesamt sind Tumoreffekte möglich, aber in ihrer Richtung und Ausprägung schwer abschätzbar.

Nach mehr als 15 Jahren Routineeinsatz gibt es aber nur sehr begrenzt Daten, die als Argument für die Förderung der Malignomentstehung unter speziellen Bedingungen dienen können. Dazu gehört primär eine erhöhte Rate von Malignomen der Haut, wobei neuere Arbeiten eher dafür sprechen, dass die Melanomrate erhöht ist, während die erhöhte Inzidenz von Non-Melanom-Hauttumoren mit der RA selbst assoziiert ist [20].
Die komplexe Datenlage zu dieser Frage wird im Artikel zu den Hauttumoren in diesem Heft näher erläutert. Zudem wurden im Wegener Granulomatosis Etanercept Trial (WGET) bei Patienten mit Granulomatose mit Polyangiitis (Wegener) unter der Kombination Etanercept und Cyclophosphamid mehr solide Malignome gefunden als unter Cyclophosphamid alleine [32]. Dieser Unterschied war statistisch signifikant. In beiden Situationen gab es gleichzeitig ein zu DNA-Schäden führendes Agens (UV-Licht bzw. Cyclophosphamid) und die Hemmung von TNF. Das wäre am ehesten mit einer fehlenden inflammatorischen Abräumung transformierter Zellen vereinbar. Sonst gibt es keine Hinweise auf eine erhöhte Inzidenz solider Malignome.

Bei (v. a. jungen männlichen) CEDPatienten treten unter der Kombination von Anti-TNF-Antikörpern mit Azathioprin hepatosplenische T-Zell-Lymphome deutlich häufiger, aber noch immer sehr selten auf [16]. Diese sonst extrem seltenen Lymphome haben bislang leider eine extrem schlechte Prognose. Sie wurden bei Patienten mit entzündlich rheumatischen Erkrankungen und unter DMARDs bisher nicht beobachtet. Das legt einen direkten Zusammenhang mit den CED und Azathioprin nahe. Sonst ist die TNF-Blockade mit einem Rückgang des bei der RA erhöhten Lymphomrisikos assoziiert, wobei aber im Einzelfall ein negativer Einfluss nicht ausgeschlossen werden kann.

\section{Fazit für die Praxis}

- Das Spektrum der Effekte des Immunsystems bei der Tumorentstehung wird in seiner Komplexität nicht überschaut.

- Während das angeborene Immunsystem die Tumorentstehung auf unterschiedliche Weise fördern kann, spielt das adaptive Immunsystem bei der Tumorabwehr eine Rolle. Dies zeigt, dass auch eine therapeutische Beeinflussung des Immunsystems sehr unterschiedliche Effekte auf die Tumorentstehung haben kann.

- Obwohl Grundlagenarbeiten in den vergangenen Jahren immunologische Signalwege identifizieren 
konnten, die an der Tumorpathogenese beteiligt sind, gibt es bisher nur wenige klinische Daten, die konkrete Hinweise für ein verändertes Malignomrisiko bei einer Therapie der RA belegen.

- Während unter einer Anti-TNFTherapie ein erhöhtes Melanom- und bei CED-Patienten Lymphomrisiko vorhanden sein dürfte, gibt es für die Therapie mit Rituximab, Abatacept oder Tocilizumab wenige Daten. Eine noch genauere Einschätzung des möglichen Risikos bedarf weiterer Langzeitdaten.

\section{Korrespondenzadresse}

\section{Prof. Dr. M. Aringer}

Medizinische Klinik und Poliklinik III, Universitätsklinikum Carl Gustav Carus an der TU Dresden

Fetscherstraße 74, 01307 Dresden, Deutschland Martin.aringer@uniklinikum-dresden.de

\section{Einhaltung ethischer Richtlinien}

Interessenkonflikt. Dieses Manuskript entstand auf Basis einer von Roche und Chugai unterstützten Arbeitsgruppe (Systemerkrankung RA), aber ohne jegliche Einflussnahme der Firmen auf das Ergebnis oder die Publikation. Die Autoren weisen auf folgende Beziehungen hin: M. Waldner: Referentenhonorare durch die Firmen Roche, Chugai und Takeda. A. Strangfeld gibt an, dass kein Interessenkonflikt besteht. M. Aringer: Advisory Boards AbbVie, Chugai, MSD, Novartis, Pfizer, Roche.

Dieser Beitrag beinhaltet keine Studien an Menschen oder Tieren.

\section{Literatur}

1. Ando K, Takahashi F, Motojima S, Nakashima K, Kaneko N, Hoshi K et al (2013) Possible role for tocilizumab, an anti-interleukin-6 receptor antibody, in treating cancer cachexia. J Clin Oncol 31(6):e69-e72

2. Baecklund E, Smedby KE, Sutton LA, Askling J, Rosenquist R (2014) Lymphoma development in patients with autoimmune and inflammatory disorders - what are the driving forces? Semin Cancer Biol 24:61-70

3. Castano-Milla C, Chaparro M, Gisbert JP (2014) Systematic review with meta-analysis: the declining risk of colorectal cancer in ulcerative colitis. Aliment Pharmacol Ther 39(7):645-659

4. Coussens LM, Werb Z (2002) Inflammation and cancer. Nature 420(6917):860-867

5. Dijkgraaf EM, Santegoets SJ, Reyners AK, Goedemans R, Wouters MC, Kenter GG et al (2015) A phase I trial combining carboplatin/doxorubicin with tocilizumab, an anti-IL-6R monoclonal antibody, and interferon-alpha $2 \mathrm{~b}$ in patients with recurrent epithelial ovarian cancer. Ann Oncol 26(10):2141-2149

6. Fallah M, Liu X, Ji J, Forsti A, Sundquist K, Hemminki K (2014) Hodgkin lymphoma after autoimmune diseases by age at diagnosis and histological subtype. Ann Oncol 25(7):1397-1404

7. Faurschou M, Sorensen IJ, Mellemkjaer L, Loft AG, Thomsen BS, Tvede $\mathrm{N}$ et al (2008) Malignancies in Wegener's granulomatosis: incidence and relation to cyclophosphamide therapy in a cohort of 293 patients. JRheumatol 35(1):100-105

8. Fridman WH, Pages F, Sautes-Fridman C, Galon J (2012) The immune contexture in human tumours: impact on clinical outcome. Nat Rev Cancer 12(4):298-306

9. GrivennikovSI,GretenFR, Karin M(2010) Immunity, inflammation, and cancer. Cell 140(6):883-899

10. Grulich AE, van Leeuwen MT, Falster MO, Vajdic CM (2007) Incidence of cancers in people with HIV/AIDS compared with immunosuppressed transplant recipients: a meta-analysis. Lancet 370(9581):59-67

11. Hanahan D, Weinberg RA (2000) The hallmarks of cancer. Cell 100(1):57-70

12. Hodi FS, O'Day SJ, McDermott DF, Weber RW, Sosman JA, Haanen JB et al (2010) Improved survival with ipilimumab in patients with metastatic melanoma. NEngl J Med 363(8):711-723

13. Hoeijmakers JH (2009) DNA damage, aging, and cancer. N Engl J Med 361(15):1475-1485

14. Hussain SP, Harris CC (2007) Inflammation and cancer: an ancient link with novel potentials. Int Cancer 121(11):2373-2380

15. Jansen DT, El BH, Arens R, Habets KL, Hameetman M, Huizinga TW et al (2015) Abatacept decreases disease activity in a absence of CD4(+) T cells in a collagen-induced arthritis model. Arthritis Res Ther 17(1):220

16. Kotlyar DS, Osterman MT, Diamond RH, Porter D, Blonski WC, Wasik M et al (2011) A systematic review of factors that contribute to hepatosplenic T-cell lymphoma in patients with inflammatory bowel disease. Clin Gastroenterol Hepatol 9(1):36-41

17. Mercer LK, Davies R, Galloway JB, Low A, Lunt M Dixon WG et al (2013) Risk of cancer in patients receiving non-biologic disease-modifying therapy for rheumatoid arthritis compared with the UK general population. Rheumatology 52(1):91-98

18. Mercer LK, Green AC, Galloway JB, Davies R, Lunt $M$, Dixon WG et al (2012) The influence of anti-TNF therapy upon incidence of keratinocyte skin cancer in patients with rheumatoid arthritis: Iongitudinal results from the british society for rheumatology biologics register. Ann Rheum Dis 71(6):869-874

19. Parodi S, Santi I, Marani E, Casella C, Puppo A, Sola $S$ et al (2015) Chronic diseases, medical history and familial cancer, and risk of leukemia and non-Hodgkin's lymphoma in an adult population: a case-control study. Cancer Causes Control 26(7):993-1002

20. Raaschou P, Simard JF, Holmqvist M, Askling J (2013) Rheumatoid arthritis, anti-tumour necrosis factor therapy, and risk of malignant melanoma: nationwide population based prospective cohort study from Sweden. BMJ 346:f1939

21. Ruperto N, Lovell DJ, Quartier P, Paz E, Rubio-Perez $\mathrm{N}$, Silva CA et al (2008) Abatacept in children with juvenile idiopathic arthritis: a randomised, doubleblind, placebo-controlled withdrawal trial. Lancet 372(9636):383-391

22. Schiff $M$, Weinblatt $M E$, Valente $R$, van der Heijde D, Citera G, Elegbe A et al (2014) Head-to-head comparison of subcutaneous abatacept versus adalimumab for rheumatoid arthritis: two-year efficacy and safety findings from AMPLE trial. Ann Rheum Dis 73(1):86-94

23. Schutz N, Marker-Hermann E (2012) Rheumatoid arthritis and multiple myeloma as comorbidity. Is tocilizumab a therapy option? Z Rheumatol 71(1):78-82

24. Shimada H, Dobashi $H$, Morimoto $H$, Kameda T, Susaki K, Izumikawa M et al (2015) Primary central nervous system lymphoma in a rheumatoid arthritis patient treated with methotrexate: a case report. BMC Res Notes 8:88

25. Simon TA, Smitten AL, Franklin J, Askling J, Lacaille D, Wolfe F et al (2009) Malignancies in the rheumatoid arthritis abatacept clinical development programme: an epidemiological assessment. Ann Rheum Dis 68(12):1819-1826

26. Simon TA, Thompson A, Gandhi KK, Hochberg MC, Suissa S (2015) Incidence of malignancy in adult patients with rheumatoid arthritis: a metaanalysis. Arthritis Res Ther 17(1):212

27. Strangfeld A, Pattloch D, Herzer P, Edelmann E, Zinke S, Aringer M et al (2013) Risk of cancer recurrence or new tumors in RA patients with prior malignancies treated with various biological agents. Arthritis Rheum 65(10):342

28. Teng MW, Swann JB, Koebel CM, Schreiber RD, Smyth MJ (2008) Immune-mediated dormancy: an equilibrium with cancer. J Leukoc Biol 84(4):988-993

29. Thomas-Tikhonenko A, Hunter CA (2003) Infection and cancer: the common vein. Cytokine Growth FactorRev 14(1):67-77

30. Topalian SL, Drake CG, Pardoll DM (2015) Immune checkpoint blockade: a common denominator approach to cancer therapy. Cancer Cell 27(4):450-461

31. Waldner MJ, Neurath MF (2015) Mechanisms of immune signaling in colitis-associated cancer. Cell Mol Gastroenterol Hepatol 1(1):6-16

32. Wegener's Granulomatosis Etanercept Trial (WGET) Research Group (2005) Etanercept plus standard therapy for Wegener's granulomatosis. N Engl J Med 352(4):351-361

33. Weinblatt ME, Moreland LW, Westhovens R, Cohen RB, Kelly SM, Khan N etal (2013) Safety of abatacept administered intravenously in treatment of rheumatoid arthritis: integrated analyses of up to 8 years of treatment from the abatacept clinical trial program. JRheumatol 40(6):787-797

34. Zhang Y, Morgan R, Podack ER, Rosenblatt J (2013) $B$ cell regulation of anti-tumor immune response. Immunol Res 57(1-3):115-124 